\title{
COVID-19 and endocrine diseases. A statement from the European Society of Endocrinology
}

\author{
M. Puig-Domingo ${ }^{1} \cdot$ M. Marazuela ${ }^{2} \cdot$ A. Giustina ${ }^{3,4}$ \\ Keywords Covid-19 $\cdot$ Diabetes mellitus $\cdot$ Obesity $\cdot$ Malnourishment $\cdot$ Hypoadrenalism
}

Published online: 11 April 2020

(c) Springer Science+Business Media, LLC, part of Springer Nature 2020

\section{Introduction}

Coronavirus disease 2019 (COVID-19) outbreak requires that endocrinologists from all over Europe move on, even more, to the first line of care of our patients, also in collaboration with other physicians such as those in internal medicine and emergency units. This will preserve the health status and prevent the adverse COVID-19-related outcomes in people affected by different endocrine diseases. People with diabetes in particular are among those in high-risk categories who can have serious illness if they get the virus, according to the data published so far from the Chinese researchers, but other endocrine diseases such as obesity, malnutrition, and adrenal insufficiency may also be impacted by COVID-19. Therefore, since the responsibilities of endocrinologists worldwide due to the current COVID-19 outbreak are not minor we have been appointed by the European Society of Endocrinology (ESE) to write the current statement in order to support the ESE members and the whole endocrine community in this critical situation.

In addition, endocrinologists, as any other healthcare worker under the current COVID-19 outbreak, will need to

A. Giustina

giustina.andrea@hsr.it

1 Endocrinology and Nutrition Service, Department of Medicine, Germans Trias i Pujol Health Science Research Institute and Hospital, Universitat Autònoma de Barcelona, Badalona, Spain

2 Department of Endocrinology and Nutrition, Hospital Universitario de la Princesa, Instituto de Investigación Princesa, Universidad Autónoma de Madrid, Madrid, Spain

3 Chair of Endocrinology, Vita-Salute San Raffaele University, Milan, Italy

4 Division of Endocrinology, IRCCS San Raffaele Hospital, Milan, Italy self-protect from this viral disease, which is demonstrating to have a very high disseminating and devastating capacity. We urge Health Authorities to provide adequate protection to the whole workforce of health professionals and to consistently test for COVID-19 the exposed personnel. A decrease in the number of healthcare professionals available for active medical practice in case they contract the disease as it is happening in certain countries, is itself, a threat for the healthcare system and the well-being of our patients.

The virus seems to have spread from infected animals and human-to-human transmission is now more than evident, with a high suspicion that non-symptomatic individuals act as the major vectors. It spreads like any other respiratory infectious disease, through contaminated airdroplets that come out of the mouth of infected persons when talking, coughing, or sneezing. The virus can survive in the environment from a few hours to a few days, depending on surfaces and environmental conditions, and touching affected surfaces. The mouth, nose, and ocular mucosa appears to be the major way of transmission.

\section{Symptoms of COVID-19 infection}

General symptoms are relatively nonspecific and similar to other common viral infections targeting the respiratory system, and include fever, cough, myalgia, and shortness of breath. The clinical spectrum of the virus ranges from mild disease with nonspecific signs and symptoms of acute respiratory illness, to severe pneumonia with respiratory failure and septic shock. Possibly, an overreaction of the immune system leading to an autoimmune aggression of the lungs could be involved in the most severe cases of acute distress respiratory syndrome. There have also been reports of asymptomatic infection and research in this matter is currently ongoing worldwide to elucidate the real prevalence of the disease and the true relative mortality ratio. 


\section{COVID-19 infection and diabetes mellitus}

\section{Increased risk of morbidity and mortality in patients with diabetes regarding COVID-19 infection}

Older adults and those with serious chronic medical conditions like heart disease, lung disease, and diabetes are at the highest risk for complications from COVID-19 infection. Chronic hyperglycemia negatively affects immune function and increases the risk of morbidity and mortality due to any infection and is associated to organic complications. This is also the case for COVID-19 infection [1]. During the Influenza A (H1N1) pandemic, the presence of diabetes tripled the risk of hospitalization and quadrupled the risk of ICU admission once hospitalized. Among COVID-19 mortality cases in Wuhan, China, major associated comorbidities included hypertension (53.8\%), diabetes $(42.3 \%)$, previous heart disease $(19.2 \%)$, and cerebral infarction (15.4\%) [2]. In addition, as for seasonal influenza, new data regarding COVID-19 indicate that the infection potentiates myocardial damage and identifies underlying heart disorders as a new risk factor for severe complications and worsening of prognosis [3].

Among the confirmed COVID-19 cases in China by Feb 11,2020 , the overall mortality reported is $2.3 \%$ [4]. This data refers mostly to hospitalized patients $[4,5]$. Among persons with no underlying medical conditions, the reported mortality in China is $0.9 \%$.

There is a lack of data regarding the number of nonsymptomatic cases, as in most countries universal microbiological screening has not been performed. It is presumed that the prevalence of the infection is probably high or very high in the community, thus leading to an overestimation of the prevalence of case fatality. However, mortality is strongly increased with the presence of comorbid diseases, including previous cardiovascular disease (10.5\%), diabetes (7.3\%), chronic respiratory disease, hypertension, and cancer, each at 6\%. Among 60-year-old people and older, mortality has been reported to be $14.8 \%$ in those $>80$ years, $8 \%$ for those between 70 and 79 years and $3.6 \%$ in the group of 60-69 years. Compared with non-ICU patients, critically ill patients are older (median age 66 vs. 51 years) and have more previous comorbidities (72\% vs. 37\%) [6]. Worldwide mortality rates may vary by region, but this information is not yet consistently available and comparable, as public health policies applied and health registers used in every region of the world are not homogeneous.

\section{What people with diabetes should do to prevent infection by COVID-19}

Social distancing as well as home confinement of the whole population are now widely adopted in many countries in
Europe and worldwide as measures hopefully effective in contrast to the spread of infection. We recommend that due to the increased dangers of developing COVID-19, persons with diabetes should strictly adhere to these preventive measures and adopt them also within their homes in order to avoid being in contact with their relatives. Therefore, under these circumstances, it is recommended that people with diabetes try to plan ahead of time what to do in case they get ill. It is important to maintain a good glycemic control, because it might help reduce the risk of infection itself and may also modulate the severity of the clinical expression of the disease.

Contact with healthcare providers, such as endocrinologists in the case of type 1 diabetes, and including also internal medicine specialists and general practitioners for type 2 diabetes patients may be advisable. However, routine appointments in person are not recommended for people with diabetes, as they should avoid crowds (waiting rooms). Therefore, we recommend phone calls, video calls, and emails as the main way for patients to keep in touch with their healthcare provider team, in order to guarantee an optimal control of the disease. Moreover, it is advised to ensure adequate stock of medications and supplies for monitoring blood glucose during the period of home confinement.

\section{What people with diabetes should do if they are infected by COVID-19}

People with diabetes who are infected with COVID-19 may experience a deterioration of glycemic control during the illness, like in any other infectious episodes. Implementation of "Sick day rules" is therefore mandatory to overcome potential diabetes decompensation. Contacting the healthcare provider team by telephone, email, or videoconference is also mandatory in case of possible symptoms of COVID19 infection in order to seek advice concerning the measures to avoid risk of deterioration of diabetes control or the possibility to be referred to another specialist (pneumologist or infectious disease doctor) or in the Emergency Services of the referral hospital to avoid the most serious systemic complication of the viral infection itself.

\section{COVID-19 and other endocrine and metabolic disorders}

\section{Obesity}

There is a general lack of data regarding the impact of COVID -19 in people suffering from obesity. However, as for what is currently being the experience in some hospitals in Spain, cases of young people in which severe obesity is present may evolve toward destructive alveolitis with respiratory failure and death (Puig-Domingo $\mathrm{M}$, personal 
experience). There is no current explanation for this clinical presentation, although it is well known that severe obesity is associated to sleep-apnea syndrome, as well as to surfactant dysfunction, which may contribute to a worse scenario in the case of COVID-19 infection. Also, deterioration of glycemic control is associated with an impairment of ventilatory function and thus may contribute to a worse prognosis in these patients. In addition, type 2 diabetes and obesity may concur in a given patient, which typically is also frequently accompanied by an age $>65$. In summary, these patients may be at a higher risk of impaired outcomes in the case of COVID-19 infection.

\section{Undernourishment}

Regarding undernourished subjects, COVID-19 infection is associated to a high risk of malnutrition development, mostly related to increased requirements and the presence of a severe acute inflammatory status. These patients show also a hyporexic state, thus contributing to a negative nutritional balance. Estimated nutritional requirements are $25-30 \mathrm{kcal} / \mathrm{kg}$ of weight and $1.5 \mathrm{~g}$ protein $/ \mathrm{kg} /$ day [7]. A nutrient dense diet is recommended in hospitalized cases including high protein supplements (2-3 intakes per day) containing at least $18 \mathrm{~g}$ of protein per intake. Adequate supplementation of vitamin D is recommended particularly in areas with large known prevalence of hypovitaminosis D and due to the decreased sun exposure $[8,9]$. If nutritional requirements are not met, complementary or complete enteral feeding may be required, and in case that enteral feeding may not be possible due to inadequate gastrointestinal tolerance, the patient should be put on parenteral nutrition. COVID-19 patients' outcome is expected to improve with nutritional support [10].

\section{Adrenal insufficiency}

Adrenal insufficiency is a chronic condition of lack of cortisol production. Live-long replacement treatment aiming to mimic physiologic plasma cortisol concentrations is not easy for these patients.

Based on current data, there is no evidence that patients with adrenal insufficiency are at increased risk of contracting COVID-19. However, it is known that patients with Addison's disease (primary adrenal insufficiency) and congenital adrenal hyperplasia have a slightly increased overall risk of catching infections. Moreover, primary adrenal insufficiency is associated to an impaired natural immunity function with a defective action of neutrophils and natural killer cells [11]. This may explain, in part, this slightly increased rate of infectious diseases in these patients, as well as an overall increased mortality. This latter could also be accounted by an insufficient compensatory increase of the hydrocortisone dosage at the time of the beginning of an episode of infection. For all these reasons, patients with adrenal insufficiency may be at higher risk of medical complications and eventually at increased mortality risk in the case of COVID-19 infection. So far, there are no reported data on the outcomes of COVID-19 infection in adrenal insufficient subjects.

In the case of suspicion of COVID-19, a prompt modification of the replacement treatment as indicated for the "Sick days" should be established when minor symptoms appear. This means in the first instance to at least double the usual doses of glucocorticoid replacement, to avoid adrenal crisis. In addition, patients are also recommended to have sufficient stock at home of steroid pills and injections in order to maintain the social confinement that is required in most of the countries for impeding the COVID-19 outbreak spread.

\section{Actions to be taken if infection by COVID-19 is suspected}

If a person with endocrine and metabolic diseases has fever with cough or trouble breathing and may have been exposed to COVID-19 (if living in or visited a country affected in the 14 days before getting sick, or if having been around a person who may have had the virus), a call to the physician or nurse for advice should be made. Some countries have set up COVID-19 phone lines for the public. The personnel in charge of these phone lines will prioritize arrangements, if needed, regarding what should be the next step in the healthcare protocol. If the person is advised to go to the hospital, it is recommended to put on a face mask. In countries with explosive outbreak, most of the people have already bought a face mask by their own initiative. Fluid samples taken from the nose or throat will be used for microbiologic diagnosis. There is currently no specific treatment for COVID-19, but since the majority of cases are mild, only a limited amount of people will require hospitalization for supportive care. However, in most of the countries in which the outbreak has been declared and recognized, particularly in China, the Northern regions of Italy, Iran, and Spain, the situation has been very challenging and the requirement of hospitalization has led national health systems to the limit of their capacities [12].

\section{What to do in case of confinement at home?}

Individuals and families affected or suspected to be affected by COVID-19 that stay at home should follow proper measures for infection prevention and control. Management should focus on prevention of transmission to others and monitoring for clinical deterioration, which may prompt 
hospitalization. Affected persons should be placed in a wellventilated single room, while household members should stay in a different room or, if that is not possible, maintain a distance of at least one meter from the person affected (e.g., sleep in a separate bed) and perform hand hygiene (washing hands with soap and water) after any type of contact with the affected person or their immediate environment. When washing hands, it is preferable to use disposable paper towels to dry them. If these are not available, clean cloth towels should be used and replaced when wet. To contain respiratory secretions, a medical mask should be provided to the person affected and worn as much as possible. Individuals who cannot tolerate a medical mask should use rigorous respiratory hygiene-i.e., the mouth and nose should be covered with a disposable paper tissue when coughing or sneezing. Caregivers should also wear a tightly fitted medical mask that cover their mouth and nose when in the same room is present the person affected.

\section{Conclusions}

\section{An ESE "decalog" for endocrinologists in the COVID- 19 pandemic}

1. Adequately protect yourself and ask for COVID-19 testing if exposed.

2. Avoid unnecessary routine appointments in person.

3. Put in place online/email/phone consultation services.

4. Closely monitor glycemic control in patients with diabetes.

5. Recommend to persons with diabetes a strict adherence to general preventive measures.

6. Counsel persons with diabetes about specific measures related to their disease management (sick day rules) in case of infection by COVID-19.

7. Counsel persons with diabetes particularly if aged over 65 and obese about referrals for management in case of suspected infection by COVID-19.

8. Avoid undernourishment with dietary or adjunctive measures if clinically indicated.

9. Closely monitor clinical conditions of patients with adrenal insufficiency.

10. Adapt increased replacement treatment if clinically indicated in patients with adrenal insufficiency.

\section{Compliance with ethical standards}

Conflict of interest The authors declare that they have no conflict of interest.
Publisher's note Springer Nature remains neutral with regard to jurisdictional claims in published maps and institutional affiliations.

\section{References}

1. J. Casqueiro, J. Casqueiro, C. Alves, Infections in patients with diabetes mellitus: a review of pathogenesis. Indian J. Endocrinol. Metab. 16(Suppl 1), S27-S36 (2012). https://doi.org/10.4103/ 2230-8210.94253

2. S.-Q. Deng, H.-J. Peng, Characteristics of and public health responses to the coronavirus disease 2019 outbreak in China. J. Clin. Med. 9(2), 575 (2020)

3. R. Gupta, A. Ghosh, A.K. Singh, A. Misra, Clinical considerations for patients with diabetes in times of COVID-19 epidemic. Diabetes Metab. Syndr. 14, 211-212 (2020). https://doi.org/10. 1016/j.dsx.2020.03.002

4. Z. Wu, J.M. McGoogan, Characteristics of and Important Lessons From the Coronavirus Disease 2019 (COVID-19) Outbreak in China: Summary of a Report of 72314 Cases From the Chinese Center for Disease Control and Prevention. JAMA (2020). https:// doi.org/10.1001/jama.2020.2648

5. Center for Disease Control and Prevention. Interim clinical guidance for management of patients with confirmed coronavirus disease (COVID-19). (2020). https://www.cdc.gov/coronavirus/ 2019-ncov/hcp/clinical-guidance-management-patients.html\#

6. D. Wang, B. Hu, C. Hu, et al. Clinical characteristics of 138 hospitalized patients with 2019 novel coronavirus-infected pneumonia in Wuhan, China. JAMA. (2020). https://doi.org/10.1001/ja ma.2020.1585

7. R. Barazzoni, et al. ESPEN expert statements and practical guidance for nutritional management of individuals with SARS-CoV2 infection. Clin. Nutr. https://doi.org/10.1016/j.clnu.2020.03.022

8. A. Giustina, R.A. Adler, N. Binkley et al. Controversies in vitamin D: summary statement from an International Conference. J. Clin. Endocrinol. Metab. 104(2), 234-240 (2019)

9. R. Bouillon, C. Marcocci, G. Carmeliet et al. Skeletal and extraskeletal actions of vitamin D: current evidence and outstanding questions. Endocr. Rev. 40(4), 1109-1151 (2019)

10. M.D. Ballesteros, M.A. Rubio Herrera, I. Bretón, Management of disease-related malnutrition in hospitalized patients with COVID19. Statement of the nutrition section, Spanish Society of Endocrinology and Nutrition, 2020

11. I. Bancos, J. Hazeldine, V. Chortis, P. Hampson, A.E. Taylor, J. M. Lord, W. Arlt, J. Eur, Primary adrenal insufficiency is associated with impaired natural killer cell function: a potential link to increased mortality. Eur. J. Endocrinol. 176(4), 471-480 (2017). https://doi.org/10.1530/EJE-16-0969

12. Y.H. Jin, L. Cai, Z.S. Cheng, H. Cheng, T. Deng, Y.P. Fan, C. Fang, D. Huang, L.Q. Huang, Q. Huang, Y. Han, B. Hu, F. Hu, B.H. Li, Y.R. Li, K. Liang, L.K. Lin, L.S. Luo, J. Ma, L.L. Ma, Z.Y. Peng, Y.B. Pan, Z.Y. Pan, X.Q. Ren, H.M. Sun, Y. Wang, Y.Y. Wang, H. Weng, C.J. Wei, D.F. Wu, J. Xia, Y. Xiong, H. B. Xu, X.M. Yao, Y.F. Yuan, T.S. Ye, X.C. Zhang, Y.W. Zhang, Y.G. Zhang, H.M. Zhang, Y. Zhao, M.J. Zhao, H. Zi, X. T. Zeng, Y.Y. Wang, X.H. Wang; for the Zhongnan Hospital of Wuhan University Novel Coronavirus Management and Research Team, Evidence-Based Medicine Chapter of China International Exchange and Promotive Association for Medical and Health Care (CPAM), A rapid advice guideline for the diagnosis and treatment of 2019 novel coronavirus (2019$\mathrm{nCoV}$ ) infected pneumonia (standard version). Mil. Med. Res. 7 (1), 4 (2020). https://doi.org/10.1186/s40779-020-0233-6 